\title{
Clock-Drawing Test Screens for Psychiatric Patients with Judicial Background: A Computer Aided
}

\author{
Elham Foroozandeh ${ }^{1 *}$, Pouya Derakhshan-Barjoei ${ }^{2}$ and $M o j d e h ~ B a h a d o r z a d e h{ }^{3}$ \\ ${ }^{1}$ Department of Psychology, Islamic Azad University, Iran \\ ${ }^{2}$ Department of Telecommunication Engineering, Islamic Azad University, Iran \\ ${ }^{3}$ Department of General Surgery, Qom University of Medical Sciences, Iran
}

*Corresponding author: Elham Foroozandeh, Assistant Professor, Department of Psychology, Naein Branch, Islamic Azad University, Naein, Iran Submission: 眥 December 12, 2017; Published: 眥 January 24, 2018

\begin{abstract}
One of the evaluations and studies to assess the health of psychiatric patients with criminal background is to use appropriate methods. In this field, evaluation of cognitive health and monitoring of problems such as structural and functional memory and functional apraxia, and executive functions, especially for psychiatric patients, are important. The use of measurement methods that can be used to accurately assess the cognitive status of patients as quickly as possible can in practice help the specialist and therapist. In this paper, we have been focusing on this issue using the clock drawing test. Also, using an evolutionary Particle Swarm Algorithm, we conducted an assessment of systemic diagnosis by comparing healthy individuals and study cases that helped us with decision making.
\end{abstract}

Keywords: Clock drawing test; Psychiatric patients; Particle Swarm algorithm

\section{Introduction}

One of the first steps in assessing the health of psychiatric patients with a criminal history is the use of appropriate methods for evaluation and measurement. In the field of neuroscience, cognitive health assessment and monitoring of problems such as structural and functional memory and functional a praxis, and executive functions, especially for psychiatric patients, are important. The use of scalable methods that can accurately assess patients' cognitive status as quickly as possible can actually help nurses, psychologists and psychiatrists. Clock Drawing Test (CDT) is a test that, in addition to the run-time, provides accurate information on verbal perception, executive functions, semantic perception, memory, and visual-spatial coordination [1]. Problems in placing numbers in the right place, the inability to show the minutes, shifting the minutes in minutes, and the inability to figure out a longer grip, including major errors in schizophrenia patients, have been reported [2]. TEST Clocking in neurological assessments has a special place and evaluates various cognitive functions in addition to visualization. Drawing the clock based on memory and putting the dials at a specific time requires the ability to continue listening, the ability to continue drawing instructions, memorizing structures, and the ability to translate information about effective visual perceptions. These cognitive functions are often consistent with dementia [3,4]. As a result, drawing a clock is a useful tool for assessing cognitive impairment, especially in the frontal lobe. In this research, a cohort study was performed on patients who were admitted to psychiatric hospitals who had a legal record and who had committed crimes before being admitted [5].

\section{Method}

For this study, 30 patients were admitted to Isfahan Psychiatric Hospital, CDT test was performed individually. All of these patients had a history of crime and were introduced to the hospital through the Iranian judicial system. The test scoring was done based on the CLOCK scoring system (from 0 to 15 , the score increase indicates better performance). The mean age of patients was $38 \pm 7.4$ years and their education varied from illiterate to graduate (with an average number of years of study, 7 years).

In some studies, fuzzy logic has also been used to estimate and evaluate [6,7]. In this inspirational study, we conducted a survey and categorization by Particle Swarm Algorithm (PSO), an evolutionary algorithm inspired by nature. Cognitive evaluation of patients admitted to psychiatric hospitals who have a legal record and who have committed crimes before being admitted, using a clock drawing test, and also using a particle swarm algorithm, a category of criteria and their relationship were performed. The particle swarm optimization algorithm was inspired by the behavior and movement of birds, bees and fish by Kennedy \& Eberhart [8]. This algorithm follows the conventional method of computational evolution. 
A: With random populations, possible answers begin.

B: By updating the generations, it searches for the optimal answer.

C: The population evaluation is based on previous generations.

The algorithm of optimizing the congestion of particles The probable solutions (particles) in the solution space of the problem are displaced by looking for the optimal current particles. This transition is effected by a fitness function that evaluates the quality of each particle. If the search space is D-dimensional then the position of the ammunition of the particle of the category can be represented as a D-dimensional vector:

$\mathrm{X}_{\mathrm{i}}=\left(\mathrm{X}_{\mathrm{i} 1}, \mathrm{X}_{\mathrm{i} 2}, \ldots, \mathrm{X}_{\mathrm{iD}}\right)$ Also, the particle velocity (position change) is also represented by another $\mathrm{D}$-dimensional vector Can be

\section{Findings}

Table 1: Patients' errors in the clock scoring system for the CDT test.

\begin{tabular}{|c|c|c|}
\hline Items & Points/Scores & Error Frequency \\
\hline The shape is similar to the clock & 1 & \\
\hline Clock circumference & 1 & \\
\hline Diameter 1 inch $(2.54 \mathrm{~cm})$ & 1 & \\
\hline All numbers are in the circle & 1 & * \\
\hline First, place 12.6.3.9 & 1 & * \\
\hline $\begin{array}{l}\text { The placement is correct (symmetry on both sides of the axis } \\
6-12 \text { ). If correct, the next one not done }\end{array}$ & 2 & \\
\hline If there is an incorrect error, correct or delete it & 1 & \\
\hline English numbering or just Persian has been graded & 1 & \\
\hline Sequence $12-1$ is correct, There is no drop-out or surplus & 1 & * \\
\hline There are only 2 pointers & 1 & \\
\hline Both pointers are shown in arrows & 1 & \\
\hline The hour pointer is between 1 and 12 & 1 & * \\
\hline the minute pointer is longer than the clock & 1 & * \\
\hline $\begin{array}{l}\text { None of the following: } \\
\text { 1) Arrows } 4 \text { or } 5 \\
\text { 2) Writes as } 1: 45 \text { (digital) } \\
\text { 3) Face or hand images } \\
\text { 4) Letter, word or image } \\
\text { 5) Rape, There is a roundabout (page) }\end{array}$ & 1 & \\
\hline
\end{tabular}

Based on the results of this study, the mean scores of patients in the clock drawing test were 6.53 and the most errors were plotted in accordance with the results of Table 1 for the symmetric placement of numbers at the time of the clock, the setting of the clockwise drawing and the different drawing Minutes and Hours displayed: $\mathrm{V}_{\mathrm{i}}=\left(\mathrm{V}_{\mathrm{i} 1}, \mathrm{~V}_{\mathrm{i} 2}, \ldots, \mathrm{V}_{\mathrm{iD}}\right)$. The best fit for an amine particle and its corresponding position are displayed with xpbest $_{\mathrm{i}}$ and xgbest $_{\mathrm{i}^{\prime}}$ respectively.

$$
\begin{gathered}
V_{i}(t+1)=\omega V_{i}(t)+c_{1} r_{1}\left(\operatorname{xpbest}_{i}(t)_{-} X_{i}(t)\right)+c_{2} r_{2}\left(\operatorname{xgbest}_{i}(t)_{-} X_{i}(t)\right) \\
X_{i}(t+1)=X_{i}(t)+V_{i}(t+1)
\end{gathered}
$$

The optimality of the best particle in the category (Optimal Global) should also be stored in the memory of the algorithm. The two distinguished set have been prepared. The First set of data consists of number of drawings by healthy people, and the other set contains drawings from our mentioned patient's drawings to simulation the evaluation analysis shows an improved interpretation of the proposed system in comparison with the classical ones. were longitudinal (Figure 1). The level of education of the patient showed a significant positive correlation with his scores in the hour drawing test $(r=0.66, p<0.05)$. Therefore, with increasing level of education in patients, performance scores improved in the clock drawing test. 


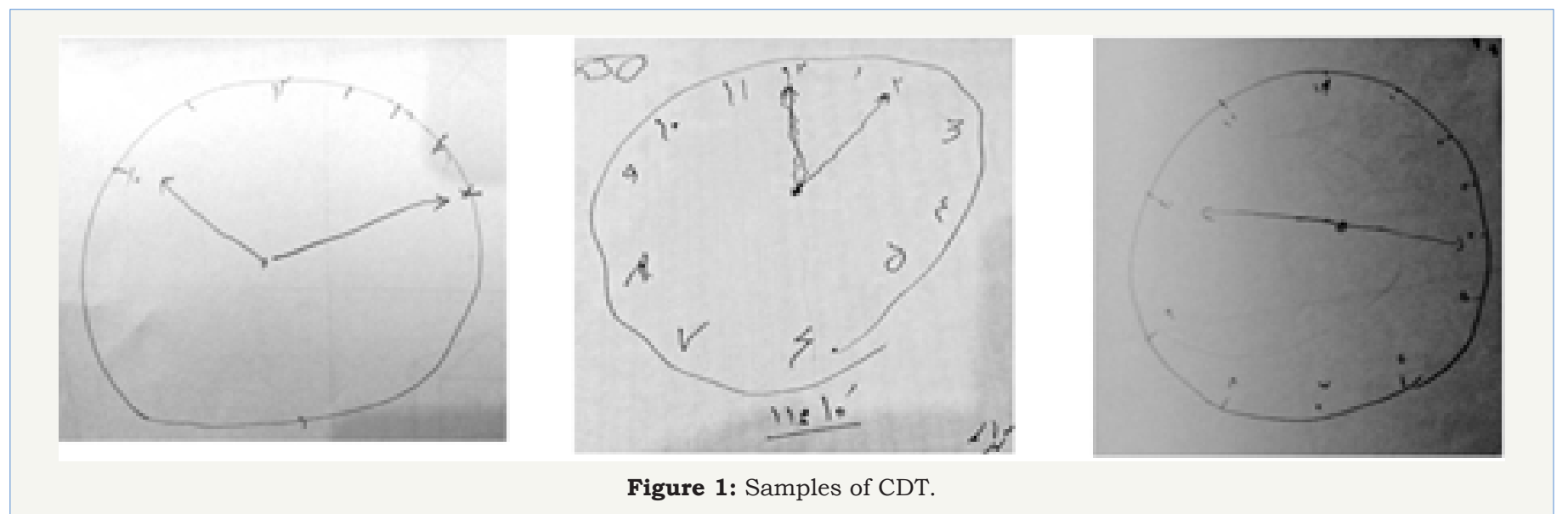

\section{Discussion and Conclusion}

In this study, although patients with criminal records and at the same time had mood disorders and thinking abnormalities, similar errors were observed with Gohari et al. (2008). According to a study conducted by Gomari Givi et al. (2008), the implementation of CDT in patients with schizophrenia in Iran was more misleading than the control group in correct placement. Approximately 78\% of the patients had a numerical placement error of about $10 \%$ of the control group. The comparison of the mean of the two groups indicates that patients with schizophrenia were significantly weaker than the control group in plotting and copying it. The hourglass test is essentially a visual-spatial assignment and is therefore sensitive to lumbar lobe damage to the right. Also, the results of this test have shown correlation with the performance of the frontal lobe, especially the executive functions [5]. According to the results of this study, it can be concluded that the problems of patients admitted to the psychiatric hospital in CDT can be related to the degradation or reduction of the ability of patients with mood disorders or thinking. Therefore, in order to quickly evaluate the neuropsychological abilities of these patients, the use of this test in psychosocial and psychological studies is recommended.

\section{References}

1. Taghi SM, Fakhrasadat GR (2014) Clock drawing test: screening of cognitive dysfunction in patients with multiple sclerosis. Journal of Isfahan Medical School 31(268): 2216-2227.
2. Hossein GG, Somayeh G (2008) The role of clock drawing test in evaluation of cortical disorder in schizophrenia. J Ardabil Univ Med Sci 8(3): 301-308.

3. Blair M, Kertesz A, McMonagle P (2006) Quantitative and qualitative analyses of clock-drawing in frontotemporal dementia and alzheimer's disease. J Int Neuropsychol Soc 12(2): 159-165.

4. Kaneda A, Yasui-Furukori N, Umeda T, Sugawara N, Tsuchimine S, et al. (2010) Comparing the influences of age and disease on distortion in the clock drawing test in japanese patients with schizophrenia. The American Journal of Geriatric Psychiatry 18(10): 908-916.

5. Bozikas VP, Kosmidis MH, Kourtis A, Gamvrula K, Melissidis P, etal. (2003) Clock drawing test in institutionalized patients with schizophrenia compared with Alzheimer's disease patients. Schizophrenia Research 59(2-3): 173-179.

6. Shigemori T, Harbi Z, Kawanaka H, Hicks Y, Tsuruoka S (2015) Feature extraction method for clock drawing test. Procedia Computer Sci 60: 1707-1714.

7. Harbi Z, Hicks Y, Setchi R (2017) Clock drawing test interpretation system. Procedia Computer Sci 112: 1641-1650.

8. Kennedy J, Eberhart R (1995) Particle swarm optimization. In Proceedings of IEEE international conference on neural networks 4(2): 1942-1948. 